\title{
II. \\ Ueber die Verwendung von Papyrus und Pergament für die juristische Litteratur der Römer.
}

\author{
Von \\ Herrn Professor Paul Krüger \\ in Königsberg.
}

Die Forschungen über die äussere Gestaltung der Schriften des Alterthums haben auch auf die juristische Litteratur der Röıner Rücksicht genommen und manche für uns werthvolle Aufklärung gebracht ${ }^{1}$ ). Es soll hier versucht werden, das für diese Untersuchungen erhaltene Material in grösserer Vollständigkeit zusammenzutragen, als es bisher verwerthet worden.

Die Schriften der römischen Juristen waren für den Rechtsunterricht und die Praxis bestimmt, wurden also regelmässig in grösseren Auflagen herausgegeben. Bis in das dritte Jahrhundert n. Chr. sind die Ausgaben auf Papyrusrollen veranstaltet worden; wenn die Membranae des Neratius wirklich nach dem zur Veröffentlichung benutzten Material benannt worden, so bildeten sie eine vereinzelte Ausnahme ${ }^{2}$ ). Die

1) Vergl. insbesondere Th. Birt das antike Buchwesen im Verhältniss zur Litteratur. 1882.

2) Die Vermuthung von Birt S. 93 f., dass Membrauae von Neratius als ein bescheidener Titel gewählt worden im Sinne von Nolizen

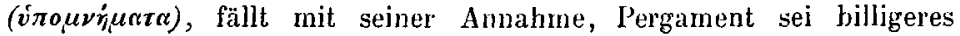
Schreibmaterial gewesen als Papyrus und deshalh zu Notizen verwendet worden; vergl. dagegen Friedländer in der Ausgabe des Martial 2 S. $299 \mathrm{f}$. 
grösseren Werke zerfielen in mehrere Rollen oder Libri, deren Umfang nicht durch die Willkür des Schriftstellers oder der Herausgeber bestimmt wurde, sondern sich dem für wissenschaftliche Schriften überhaupt üblichen Maass anschloss. Es musste dabei Rücksicht genommen werden auf die Bequemlichkeit der Leser und auf die Haltbarkeit der Rollen. Beides bedingte einen nicht allzu grossen Umfang der letzteren; andererseits hätten wiederum gar zu kleine Rollen die Benutzung grösserer Werke wesentlich erschwert. So stellte sich die Einhaltung eines gewissen Mittelmaasses fest, welches für die Schriften in Prosa zwischen 1500-2500 Zeilen von je etwa 34 Buchstaben geschwankt hat ${ }^{1}$ ).

Von den selbständig überlieferten Werken der römischen Juristen ist bekanntlich nur eines vollständig genug erhalten, um die für die übrige Litteratur gefundenen Resultate damit vergleichen zu können, die Institutionen von Gaius. Legt man die Veroneser Abschrift derselben zu Grunde ${ }^{2}$ ), so erhält man als ungefähres Mass für Buch I 1400, für Buch II 1850, für Buch III 1600, für Buch IV 1900 Normalzeilen. Doch ist die Grundlage dieser Berechnung insofern unzulänglich, als in dieser Handschrift vielfach das juristische Abkürzungssystem benutzt ist, und zwar sicher nicht etwa in einer der ursprünglichen Herausgabe des Werkes entsprechenden Weise, sondern offensichtlich nach dem Belieben der Schreiber dieser Handschrift. Dürften wir annehmen, dass die erste Ausgabe abgesehen von den Formeln, welche entsprechend den Abkürzungen in Leges und Edicta mit Litterae singulares geschrieben wurden, ohne Benutzung der Notae iuris erfolgt wäre, so würden die obigen Zeilenzahlen höchstens um 1/7 zu erhöhen sein $^{3}$ ) und fielen auch dann noch in die Grenzen des angegebenen Mittelmasses.

1) Birt S. $307 \mathrm{f}$.

$\left.{ }^{2}\right)$ Die Lücken der Veroneser Handschrift machen für die Berechnung des vollständigen Werks keine Schwierigkeit.

$\left.{ }^{3}\right)$ So z. B. sind auf Seite 38 des Veronensis ausgeschrieben 848 Buchstaben, durch Abkürzung weggelassen 84, auf S. 90 ausgeschrieben 872 , weggelassen 207 , auf S. 95 ausgeschrieben 883 , weggelassen 112 , auf S. 224 ausgeschrieben 923, weggelassen 234. Das Verhältniss der weggelassenen $\mathrm{zu}$ den ausgeschriebenen Buchstaben schwankt also 
Wir sind nun aber für die Bestimmung der Buchgrösse nicht auf die Institutionen von Gaius beschränkt. Die Justinianischen Auszüge aus der klassischen Litteratur, welche im allgemeinen nur winzige Reste der einzelnen Bücher wiedergeben, haben Ulpians Kommentar zum Edikt zur Grundlage genommen und wenigstens einige Bücher desselben so stark benutzt, dass sich dem ursprünglichen Umfang ziemlich nahe kommen lässt. So betragen die Bruchstücke von Buch 71 etwa 36800 Buchstaben, also 1082 Normalzeilen, von Buch 11 etwa 41320 Buchstaben $=1215$ Zeilen, von Buch 29 etwa 54930 Buchstaben $=1615$ Zeilen, von Buch 1 ad edictum aedilium curulium 31120 Buchstaben $=915$ Zeilen. Das 29. Buch, welches in seinem jetzigen Umfange schon dem Mittelmaass der Papyrusrollen entspricht, weist, soweit zu erkennen, nur kleinere Lücken auf ${ }^{1}$ ).

Dass der Buchabschluss nicht lediglich von der Länge der Papyrusrollen bestimmt wurde, sondern zugleich einen sachlichen Abschluss bildete, verstand sich von selbst. Gerade in den Institutionen von Gajus tritt dies bekanntlich sehr scharf hervor, Buch 1 und 4 erledigen in sich je einen Hauptabschnitt, Buch 2 und 3 umfassen den zweiten Abschnitt so, dass Buch 3 eine Unterabtheilung desselben beginnt. Durch diese sachliche Eintheilung ist das ungleiche Maass der einzelnen Bücher bedingt worden. Aehnliches lässt sich für viele Schriften der klassischen Juristen wahrscheinlich machen, doch ist in einzelnen, namentlich in den grösseren Kommentaren nachweislich nicht immer darauf gehalten, dass die Hauptabschnitte, welche durch mehrere Bücher sich erstreckten, mit einem Buchabschluss zu Ende gehen. Zwar

auf diesen Probeseiten zwischen 1:10 und 1:4. Hätte der Schreiber die Abkürzungen so ausgenutzt, wie manche unserer Gaius-Kritiker in ihren Ergänzungen der noch vorhandenen Lücken voraussetzen, so würde sich das ohige Verhältniss umgekehrt haben, die Handschrift hätte aber auch eine für den Leser unleidliche Gestalt angenommen.

1) Die Kompilatoren haben einzelne Ediktsätze und sämmtliche Klagformeln, sowie die einleitenden Bemerkungen zum SC. Velleianum weggelassen; ausserdem mögen noch einzelne Auszüge Ulpians ans Julians Digesten gestrichen sein, für welche jetzt die Stellen der letzteren eingeschoben sind. 
Ueber die Verwendung von Papyrus und Pergament etc.

fiur die Ediktkommentare von Paulus und Ulpian, soweit diese solche Partes gebildet haben ${ }^{\mathbf{1}}$ ), trifft letzteres im ganzen $\mathrm{zu}^{2}$ ); folgende Partes treten in diesen deutlich heraus:

Einleitung Paulus 1-16 Ulpian 1-14.

De iudiciis Paulus 17-27 Ulpian 15-25.

De rebus creditis Paulus 28-34 Ulpian 26-32.

De re uxoria Paulus 35-37 Ulpian 33. 34.

De tutelis Paulus 38 Ulpian 35. 36.

De furtis und De operis libertorum Paulus 39

Ulpian 37. 38.

De bonorum possessionibus Paulus $41-44$

Ulpian 39-49.

De testamentis Paulus 45. 46 Ulpian 50.

Exekution Paulus 56-62 Ulpian 58-66.

De interdictis Paulus 63-68 Ulpian 67-73.

De exceptionibus Paulus 69-71 Ulpian 74-76.

De stipulationibus Paulus 72-78 Ulpian 77--81.

Dagegen in den libri ex Sabino oder ad Sabinum von Pomponius, Paulus und Ulpian beginnen und schliessen die Hauptabtheilungen bald mit den Büchern, bald innerhalb derselben ${ }^{3}$ ).

Mehrere der klassischen Schriften haben sich einen weiteren Leserkreis auf lange Zeit erhalten, trotzdem neue Werke den gleichen Stoff unter Berücksichtigung der inzwischen cingetretenen Rechtsänderungen behandelten. Die letztern, sowie die Aussprüche der späteren Juristen über dieselben Rechtsfragen mögen wohl hauptsächlich den Anlass dazu gegeben

1) Ueber Partes im allgemeinen vergl. Birt S. $34 \mathrm{f}$.

2) So zum Theil schon in den Digesta von Celsus und noch mehr in denen Julians: Einleitung B. 1-4, Iurisdictio B. 5-22, Bonorum possessio B. 23-28, Testament B. 29-31, Legate B. 32-38, Fideikommisse B. 39. 40, Interdikte B. 48. 49, Exceptionen B. 50. 51, Stipulationen B. 52-58; abgewichen ist in B. 58, wo die Iniuriae mit dem Anfang der Exekution verbunden sind.

3) So beginnen inmitten eines Buchs: Legat bei Paulus 3, Dos bei Pomponius 14 Paulus 6, Tutel hei Pomponius 16 Ulpian 36, Furtum bei Pomponius 17 Ulpian 40, Stipulation bei Paulus 11 Ulpian 45, es schliessen inmitten eines Buchs Legat bei Pomponius 8 Panlus 5, Dos bei Pomponius 16. 
haben, dass bei Erschöpfung der ersten Auflagen eine Neubearbeitung Platz griff. Eine solche vom Verfasser selbst vorgenommene Umarbeitung wird uns von Ulpians libri ad Sabinum berichtet ${ }^{1}$ ). Ausserdem kennen wir eine Reihe neuer durch jüngere Juristen veranstalteter Ausgaben, welche sich entweder auf Notae mit kritischen Bemerkungen und Nachträgen beschränkten oder auch nur einen Auszug des ursprünglichen Werkes veröffentlichten.

Zur ersteren Gruppe gehören:

Labeo's Posteriores mit Notae von Proculus und Aristo. Sabinus Ius civile(?) und ad Vitellium mit Notae von Aristo.

Cassius Ius civile mit Notac von Aristo.

Julians Digesten mit Notae von Marcellus, Scävola, Paulus.

Pomponius Regulae mit Notae von Marcellus.

Marcellus Digesten mit Notae von Scävola und Ulpian.

Scävola's Digesten mit Notae von Claudius.

Scävola's Responsa mit Notae von Claudius und Paulus.

Papinians Quaestiones mit Notae von Paulus.

Papinians Responsa mit Notae von Paulus und Ulpian.

Papinians lib. sing. de adulteriis mit Notae von Marcian.

Auszugsweise sind veröffentlicht:

Alfenus Digesta durch Paulus,

Labeo's Posteriores durch Javolenus,

Labeo's Pithana durch Paulus, ein Werk von Vitellius durch Sabinus ${ }^{2}$ ) und Paulus, ungenannte Werke von Minicius und Urseius durch Julian, ein Werk von Neratius durch Paulus ${ }^{3}$ ).

1) Cod. Iust. praef. III (c. Cordi) $\$ 3$ a. E.

2) Die Nota von Cassius zu Vitellius in Dig. 33, 7, 12 \& 27 bezieht sich wohl auf dieses Werk, nicht auf das Original.

3) Eine Umgestaltung des Textes enthalten schon solche Werke, wie Javolenus ex Posterioribus Labeonis und ex Cassio, die Werke ex Q. Mucio oder ad Q. Mucium, Paulus ad Vitellium, wohl auch die Werke ex Plautio oder ad Plautium (wenigstens Pomponius ex Plantio); die libri ex Sabino oder ad Sabinum sind selbständige Werke, welche nur das System des Sabinus zu Grunde legen. 
Die Papyrusrollen sind allmählich durch die Pergamenthandschriften aus dem Gebrauch verdrängt worden, und zwar hat der Uebergang zu Veröffentlichungen in Pergamentcodices bei den juristischen Schriften früher stattgefunden als bei den meisten übrigen Gattungen von Schriftwerken; bei jenen fällt er schon in das 3. Jahrhundert n. Chr. Ulpian setzt noch als das gewöhnliche Buch die Rolle, das volumen, voraus ${ }^{1}$; bei der er neben Papyrus auch Verwendung von Pergament und Lindenbast erwähnt ${ }^{2}$ ); Rollen von Pergament und Bast haben aber zweifellos eine seltene Ausnahme gebildet. Dass Ulpian die Frage aufwirft, ob auch Codices von Pergament, Papyrus oder sonstigem Material unter dem Vermächtniss von libri einbegriffen seien, zeigt klar, dass diese für Schriftwerke nur selten zur Verwendung kamen ${ }^{3}$ ). Andererseits bemerkt schon Paulus ${ }^{4}$ ), dass ein Legat von libri nicht lediglich auf eine entsprechende Anzahl von Papyrusrollen gehe, sondern überhaupt nach dem feststehenden Abschluss der Schriftwerke bemessen werde; darin liegt also, dass, wenn die im Nachlass befindlichen Codices mehrere Bücher umfassten, sie nicht als nur je ein Buch in Anrechnung kämen ${ }^{5}$ ).

Die ersten Publikationen als Codices, welche wir kennen, sind die Konstitutionensammlungen, welche von dieser Gestalt auch ihre offizielle Bezeichnung entlehnten; als älteste der Codex Gregorianus, welcher unter Diokletian verfasst ist. Für die Form der Herausgabe entschied wohl nicht allein die Haltbarkeit des Pergaments gegenüber dem Papyrus, sondern auch, dass sie Nachschlagewerke waren, deren Benutzung durch die Rollenform wesentlich erschwert worden wäre. In den Codices liess sich ein ungleich grösserer Stoff unter-

1) Dig. 32, 52 pr. $\S 1$.

2) Vergl. Paul. 3, 6, 87 (Note 4).

3) Vergl. noch Birt S. $98 \mathrm{f}$.

4) Sent. 3, 6, 87: Libris legatis tam chartae volumina vel membranae et philyrae continentur: codices quoque debentur; librorum enim appellatione non volumina chartarum, sed scripturae morlus qui certo fine concluditur aestimatus:

5) Anders entscheidet Ulpian in Dig. 32, 52 $\$ 1$ für den Fall, wo im Nachlass eine Rolle sich findet, welche den ganzen Homer umfasst; diese könne der Erhe dem Legatar, dem 100 Bücher vermacht sind. nicht als 48 Bïcher, sondern nur als ein Buch geben.

Zeltschrift für Rechtsgeschlchte. VIII. Rom. Abtl. 
bringen als in den Rollen; die Bezeichnung der einzelnen Sammlung als Codex lässt darauf schliessen, dass das ganze Werk in einen Band zusammengefasst werden sollte ${ }^{1}$ ). Darum wurde aber die frühere Bucheintheilung in den grösseren Werken nicht aufgegeben, sondern als Unterabtheilung ebenso auf die neueren Werke übernommen, wie sie für die älteren bei der Uebertragung auf Codices nach der früheren Rolleneintheilung festgehalten wurde. Nur insoweit hat die Bucheintheilung eine Umgestaltung erlitten, als die Befreiung von dem Rollenumfang eine grössere Willkür in dem Mass der einzelnen. Bücher veranlasst hat. So muss der Codex Hermogenianus, welcher als Monobiblos erschien, weit über das frühere Mittelmaass der Bücher hinausgegangen sein. Beim Theodosianus schwanken die vollständig erhaltenen Bücher zwischen 1500 und 3500 Normalzeilen ${ }^{2}$ ), ebenso in Justinians Institutionen ${ }^{3}$ ). In Justinians Digesten finden wir Bücher mit 1127 (Buch 25) bis zu 5480 (Buch 50) solcher Zeilen ${ }^{4}$ ), im Justinianischen Codex ist das ursprüngliche Mittelmass ganz aufgegeben, die Bücher enthalten 4500 bis 11700 Normalzeilen ${ }^{5}$ ).

Aus der Publikation auf Rollen stammen auch die Inhaltsangaben der einzelnen Bücher, die Indices titulorum, welche ausserhalb der einzelnen geschlossenen Rolle angebracht waren. In den Codices wurde verständiger Weise aus diesen Indices ein Gesammtverzeichniss aller Bücher hergestellt und an den Anfang der Handschrift gesetzt. Gleiche Indices titulorum sind den neueren Rechtssammlungen voraufgeschickt

1) Beim Hermogenianus mag dies keine praktischen Schwierigkeiten gemacht haben; auch der Theodosianus liess sich bei grösserem Format in einen mässig dicken Band bringen; ob auch der Gregorianus, ist nicht sicher zu beantworten.

2) Buch $6=3100$, B. $7=2500$, B. $8=3000$, B. 9 u. $16=$ 3500 , B. $10=2000$, B. $11=4300$, B. $12=3650$, B. $13=1750$, B. $14=1550$, B. $15=1500$. Die in den älteren Handschriften enthaltenen Abkürzungen in den Inscriptionen und Subscriptionen sind bei dieser Berechnung nicht aufgelöst worden; sie können auch in der Original-Ausgabe schon in ähnlicher Weise gestanden haben.

3) Buch $1=1500$, B. $2=3100$, B. $3=2600$, B. $4=2300$.

4) So nach der Berechnung von Birt S. 335 .

5) Die kürzesten Bücher sind 2. 3. 11, das erste ist das längste. 
worden; doch finden sich in einzelnen. Handschriften des Theodosianus, der Lex Romana Wisigothorum und der Justinianischen Rechtsbücher auch noch Indices zu den einzelnen Büchern.

Die Zeilenlänge der früheren Zeit richtete sich zunächst nach dem Format der einzelnen zur Rolle zusammengeklebten Papyrusblätter (paginac), welche der Haltbarkeit wegen nicht über ein gewisses Mass hergestellt wurden ${ }^{1}$ ). Diese Beschränkung fiel beim Pergament weg, dafür nahm man aber jetzt Rücksicht darauf, dass nicht wie bei einzelnen Inschriften durch die Länge der Zeilen der Uebergang des Lesers von einer Zeile zur anderen und damit die Benutzung der Handschrift überhaupt erschwert werde. Man ging deshalb im allgemeinen nicht weit über das bisher übliche Mass hinaus, oder wählte sogar noch kürzere Zeilen und nutzte, wo nöthig, die Breite des Pergaments dadurch aus, dass man die Seiten in 2 oder mehr Kolumnen eintheilte. Die juristischen Codices in Unzialschrift (etwa 5.-7. Jahrhundert) haben folgende Zeilenmasse. Der alten Normalzeile entsprechen die Neapolitaner Digestenfragmente ${ }^{2}$ ) und die Veroneser Fragmente der Justinianischen Institutionen (34 B.). Ueber dieses Mass gehen hinaus die Veroneser Handschrift von Gajus mit 42 Buchstaben, der Turiner Palimpsest des Theodosianus ebenfalls mit 42 B., die Vatikanische Handschrift von Buch 9 bis 16 des Theodosianus mit 38-39 B. und die Parisèr Handschrift von Buch 6-8 des Theodosianus mit 42 B., endlich noch die Vaticana fragmenta mit 57-60 Buchstaben auf der Zeile. Kürzere Zeilen mit Kolumneneintheilung haben die Fragmente de iudiciis (15-16 B.), de iure fisci (17 B.), die Papiniansfragmente (23-29 B.), der Veroneser Codex Justinianus $(27-28$ B.) und die Florentiner Digesten $(31-32$ B.); kurze Zeilen ohne Kolumnenabtheilung finden sich in dem Vatikanischen Palimpsest des Theodosianus $(24 \text { B. })^{3}$ ), in der Münchener Handschrift der Lex Romana Wisigothorum (30 B.) und in den Papyrushandschriften der Ulpianischen Institutionen

1) Vergl. Birt S. $247 \mathrm{f}$.

$\left.{ }^{2}\right)$ Digesta ed. Mommsen I additamenta S. $3 * \mathrm{f}$.

$\left.{ }^{3}\right)$ Die dazu gehörigen Turiner Blätter sind verloren. 
(22 B.), der Sinai-Scholien zu Ulpian $(30-31$ B.) und der Pommersfeldner Digestenfragmente $(30$ B.).

Aus dieser Zusammenstellung möchte man schliessen, dass die Normalzeile nur mehr zufällig in einzelnen Codices vorkomme. Dass dies nicht zutrifft, dafür haben wir ein entscheidendes Zeugniss in Justinians Digesten. Sie machten nach Justinians eigenem Bericht etwa 150000 Zeilen aus ${ }^{1}$ ), was gegenüber der Zahl der Buchstaben eine Zeilenlänge von durchschnittlich 34 Buchstaben ergiebt ${ }^{2}$ ). Diese Angabe kann nicht auf ein einzelnes Exemplar bezogen werden, sie setzt voraus, dass die ganze ursprüngliche Ausgabe stichometrisch, also in gleicher Seiten- und Zeilenabtheilung hergestellt wurde. Wir erfahren ferner aus der Bemerkung Justinians ${ }^{3}$ ), die ausgezogenen Werke der klassischen Juristen hätten zusammen mehr als 3 Millionen Zeilen enthalten, und aus der Subscriptio des Index auctorm zu den Digesten, welche die jetzt verlorne genaue Stichenzahl angab, lässt sich schliessen, dass in den Handschriften dieser Werke sich noch ein Vermerk über die Gesammtzeilenzahl fand, wie solche vordem in den Papyrusrollen üblich waren und zum Theil auch in den Codices der nichtjuristischen Litteratur festgehalten wurden ${ }^{4}$ ). Vielleicht haben sich aber diese Handschriften nicht auf den Vermerk über die Zeilenzahl der Original-Ausgabe beschränkt; die neu gefundenen Sinai-Scholien zu Ulpian ergeben, dass wenigstens die in den Rechtsschulen noch gebrauchten Werke gerarlezu stichometrisch mit Zeilenzählung geschrieben waren. Der Scholiast setzt dies sowohl bei dem kommentirten Werk selbst als bei den sonst noch angeführten Schriften voraus. Trotzdem das erstere in Titel und Kapitel eingetheilt ist, verweist er in demselben auf 2 Blätter weiter $(14,4)$, oder lässt 100, 50, 25, 10 Zeilen überspringen $(16,65)$. 8. 17, 9. 18, 4). Ebenso verweist er in Paulus Responsa auf das

1) Dig. c. Tanta $\$ 1$ (Mommsen S. XXX*).

2) Mommsen Vorrede zu den Dig. S. XI N. 2.

3) Const. 'Tanta $\$ 1$.

4) Birt S. $162 \mathrm{f}$.

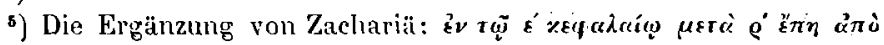

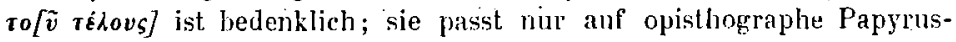
rollen, wie der Cicero des Asconius (Birt S. 176). 
2. Blatt vor dem Ende von Buch 8, in Florentins Institutionen auf das 5. Blatt vor dem Ende des 3. Buchs.

Dass die uns erhaltenen Handschriften keine stichometrischen Abschriften sind, folgt aus den Abweichungen vom Normalmaass, aus dem Mangel einer Zeilenzählung, und für die Handschriften des Theodosianus noch aus den Abweichungen derselben unter sicb. Die stichometrische $\mathrm{Ab}$ schrift der Justinianischen Kompilation wird kaum über die erste Auflage hinausgegangen sein. Eine Vorschrift sie einzuhalten war nicht gegeben, ein Bedürfniss dafür bestand auch nicht, da die Eintheilung in Titel und Fragmente oder Konstitutionen, welche dann noch in tśuava zerfielen, die Auffindung der Anführungen leicht machte. Dazu kam, dass die Scholien regelnnässig mit dem kommentirten Werk verbunden und $\mathrm{zu}$ den betreffenden Worten an den Rand gesetzt wurden, so dass eine Anführung nach Textzeilen ganz überflüssig wurde ${ }^{1}$ ). So finden wir denn auch bei den Kommentatoren des sechsten Jahrhunderts keine Benutzung stichometrischer Eintheilungen.

1) Die Verweisungen arn Rande der Vaticana fragmenta \$ 282. 294 auf Seiten der Handschrift gehören nicht zu dem Scholienapparat, sondern sind Zusätze eines Besitzers dex Handschrift und beziehen sich nur auf diese selbst. 